\title{
Photoemission studies of chiral metal surfaces using circularly polarized synchrotron radiation
}

\author{
Gary A. Attard* and David Watson \\ Department of Chemistry, University of Wales, Cardiff, P.O. Box 912, Cardiff CF10 3TB, United Kingdom \\ Elaine A. Seddon and Stephen M. Cornelius \\ CLRC Daresbury Laboratory, Daresbury, Warrington, Cheshire WA4 4AD, United Kingdom \\ Enrique Herrero and Juan Feliu \\ Department de Quimica Fisica, Universitat d'Alacant, Apartat 99, E-03080 Alacant, Spain
}

(Received 6 October 2000; published 24 August 2001)

\begin{abstract}
In an attempt to measure classical circular dichroism (CD) in the photoemission of electrons from a solid surface, valence-band photoemission from clean and adsorbate-covered chiral metal surfaces has been investigated using circularly polarized synchrotron radiation. An experimental geometry was used whereby $\vec{q}$, the polarization of the radiation, $\vec{k}$, the wave vector of the photoemitted electrons, and $\vec{n}$, the surface normal, were coplanar. It was anticipated that by doing this, so-called CDAD (circular dichroism in the angular distribution) effects associated with chiral photoemission geometries would be decoupled from classical CD arising from the difference in absorption cross-section for left and right circularly polarized light of electrons in energy states associated with $R$ and $S$ kinks. However, from the magnitude of the effects observed, it is evident that any classical $\mathrm{CD}$ features in the photoelectron spectrum arising from kinks, including photoemission from surface states or resonances, are swamped by other dichroic effects. We conclude that final-state effects in the diffraction of photoelectrons dominate the intensity of all peaks observed in angle-resolved photoemission from chiral, single crystal, metal surfaces using circularly polarized photons.
\end{abstract}

DOI: 10.1103/PhysRevB.64.115408

PACS number(s): 79.60.-i

\section{INTRODUCTION}

Single-crystal surfaces can be made chiral if they are prepared in such a way that they contain kink sites, the handedness of the kink microstructure governing the overall chirality of the crystal surface. ${ }^{1}$ It has long been argued that circular dichroism (CD), as routinely observed in photoabsorption experiments with chiral molecules, should be observed in analogous photoemission experiments. ${ }^{2,3}$ Ritchie was the first to predict theoretically that $\mathrm{CD}$ should be observed in the photoemission from gas-phase chiral molecules. ${ }^{2}$ However, he also emphasized that photoemission CD should be observed even from ostensibly nonchiral molecules so long as the they are spatially oriented. This special type of $\mathrm{CD}$ arising from the chirality of the experimental geometry (a "false" chirality, in the terminology of Barron $^{4}$ ) Ritchie called "circular dichroism in the angular distribution" (CDAD) of electrons. A substantial difference between classical $\mathrm{CD}$ and CDAD is that in the former, the effect arises from interference between electric and magnetic dipole transitions whereas, as shown by Cherepkov, ${ }^{5}$ the latter arises even in the pure electric dipole approximation. Consequently, CDAD asymmetries were predicted to exceed classical CD asymmetries by several orders of magnitude. Experimental confirmation of this prediction for surface photoemission was first presented by Westphal et al. ${ }^{6}$ in an experiment in which $\mathrm{CO}$ molecules chemisorbed on a $\operatorname{Pd}\{111\}$ substrate were oriented along the surface normal $\vec{n}$, and circularly polarized light $\vec{q}$ was utilized to generate photoemitted electrons of wave vector $\vec{k}$ in an angle-resolved experiment. So long as $\vec{n}, \vec{k}$, and $\vec{q}$ were not coplanar, large dichroic effects as a function of the angle between $\vec{k}$ and $\vec{n}$ were observed and characterized quantitatively by a CDAD asymmetry factor

$$
A_{C D A D}=\frac{I^{R}(\theta)-I^{L}(\theta)}{I^{R}(\theta)+I^{L}(\theta)} \times 100 \%,
$$

where $I^{R}(\theta)$ and $I^{L}(\theta)$ are the angle-resolved intensities of photoemission peaks using right-circularly polarized (RCP) and left circularly polarized (LCP) light respectively. The value of $A^{C D A D}$ was found to range from $70 \%$ to $-70 \%$ depending on $\theta$. With $\vec{n}, \vec{k}$, and $\vec{q}$ coplanar, the experimental $A_{C D A D}$ was found to be zero. Subsequently, CDAD was shown to be quite a general phenomenon in photoemission from clean ${ }^{7}$ and adsorbate-covered ${ }^{8}$ surfaces in both valence and core-level photoemission experiments. Recently, Ynzunza et al. ${ }^{9}$ building on earlier work by Daimon et al., ${ }^{10}$ demonstrated the importance of photoelectron diffraction in the generation of CDAD from $\mathrm{O} / \mathrm{W}\{110\}$. In particular, not only were CDAD effects as large as $+20 \%$ observed as a function of crystal azimuthal angle for normal incidence radiation and a fixed polar angle, but also the observed diffraction peaks exhibited peak "rotations" 9 across mirror planes perpendicular to the surface. The roles of single and multiple reflections and of photoelectron scattering in generating dichroic effects in photoemission at low energies (i.e., $<25$ $\mathrm{eV})$ were modeled by Fecher ${ }^{11}$ who concluded that dichroic effects are generally to be expected for both adsorbates and pure substrates (even for spherical initial states) due to the break in symmetry caused by the surface. It should be emphasized here that all of the various substrates dis- 


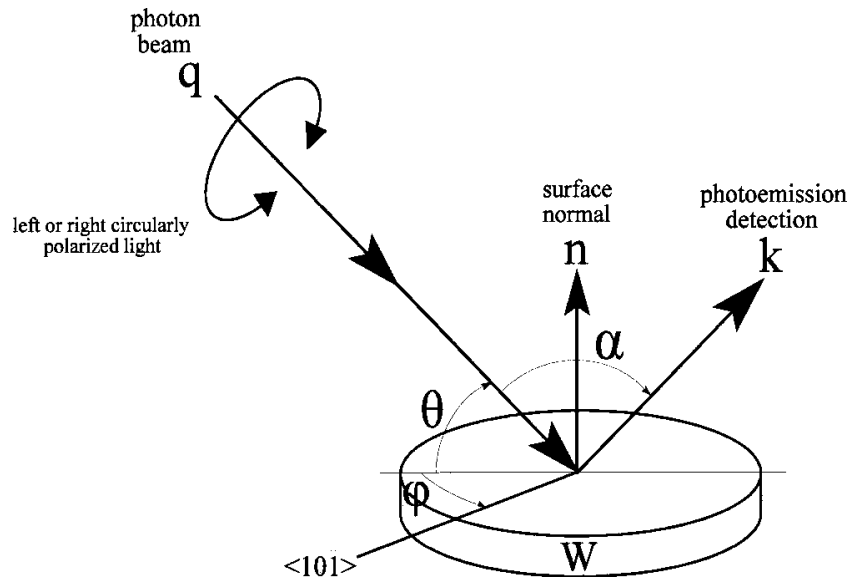

FIG. 1. Schematic diagram of the experimental geometry used in this study. $\vec{q}, \vec{n}$, and $\vec{k}$ are coplanar. $\theta$ is the photon angle of incidence, $\phi$ is the azimuthal angle and $\alpha$ is the electron detection angle with respect to the photon beam $\left(90^{\circ}\right)$.

cussed so far are nonmagnetic, and that CDAD does not require strong spin-orbit coupling in order to be observed. ${ }^{7}$ Moreover, classical CD asymmetries are normally weak (typically of the order of $1 \%$ ), and so would be swamped by CDAD asymmetries unless the latter can be decoupled from the experiment.

In an attempt to observe classical CD, we have investigated valence-band photoemission from a series of clean and adsorbate-covered chiral surfaces. The chirality of the surface originates from the microstructure of the kinks ${ }^{1}$ and both left- and right-handed kink sites have been studied and their interaction with circularly polarized UV light quantified. So long as $\vec{k}, \vec{n}$, and $\vec{q}$ are coplanar, the experimental geometry is not chiral, and it is reasonable to assume that CDAD should be absent. Any dichroic effects may then be ascribed to the asymmetry of the kinks. However, if multiple-scattering, final-state effects are significant, angulardependant intensity variations will be observed at all azimuthal angles of the crystal, since kinked single-crystal surfaces do not contain a mirror plane perpendicular to the surface. The following represents an attempt at characterizing the photoemission $\mathrm{CD}$ of an inherently chiral metal surface.

\section{EXPERIMENT}

A schematic of the experimental geometry adopted is given in Fig. 1. The tungsten crystals (Metal Crystals and Oxides Ltd.) were polished on both sides, and were held such that vectors representing the incoming photon beam, the surface normal, and the photoemission direction were coplanar. The electron take-off angle and the angle of incidence of the circularly polarized radiation from Beamline 3.3 of the SRS (Synchrotron Radiation Source) were both set to $45^{\circ}$. The precision of the crystal alignment during CDAD measurements was estimated to be within $1^{\circ}$ based on specular reflection of the visible part of the synchrotron beam by the mirror surface of the sample into the analyzer. Azimuthal precision was determined using a video low-energy electrondiffraction system and was found to be reproducible to

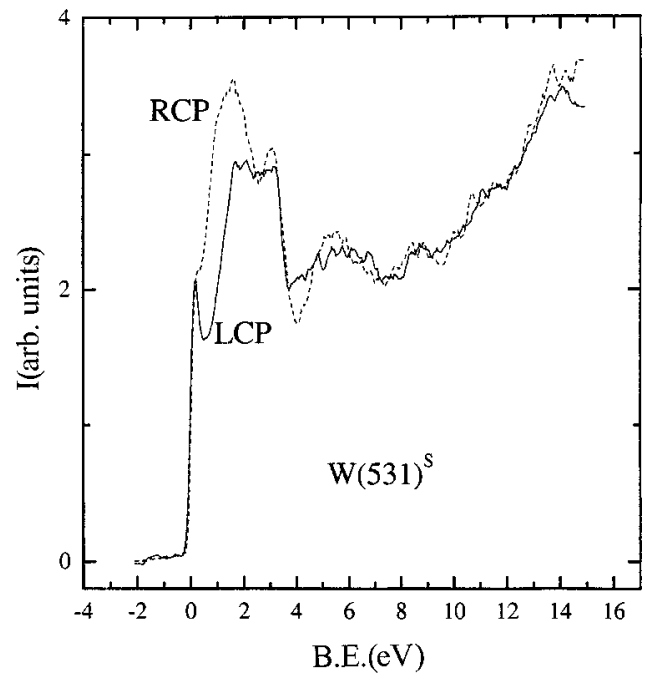

FIG. 2. Valence-band photoemission spectra of $\mathrm{W}\{531\}^{S}$ taken with right and left circularly polarized light of $30 \mathrm{eV}\left(\phi=18^{\circ}\right.$, $\theta=45^{\circ}$, and $\alpha=90^{\circ}$ ).

within $1^{\circ}$. The photoemitted electrons were energy analyzed in a VSW HA100 hemispherical deflection analyzer fitted with a Daresbury Laboratory purpose built multichannel detector system. ${ }^{12,13}$ The angular acceptance of the analyzer was approximately $\pm 3^{\circ}$. Beamline 3.3 of the SRS is equipped with a toroidal grating monochromator, the output of which has been characterized in detail. ${ }^{14}$ The circularly polarized light is selected by means of moveable baffle. At a photon energy of $30 \mathrm{eV}$, the light is approximately $75 \%$ circularly polarized. ${ }^{15}$

The base pressure in the system, with the ultrahighvacuum chamber open to the synchrotron, was 1 $\times 10^{-9}$ mbar. Hence data collection was performed immediately after flashing the crystal to $2500 \mathrm{~K}$ (while it was still cooling) in order to minimize the degree of surface contamination. The tungsten crystals were cleaned by electron-beam heating (using a tungsten filament mounted on a linear drive) in an oxygen atmosphere at $2000 \mathrm{~K}$ followed by a rapid flash to $2500 \mathrm{~K}$, in order to remove surface oxygen. The platinum crystal was prepared at Cardiff using the "Clavilier method," 16 and cleaned in $10^{-6}$ mbar of oxygen at $1000 \mathrm{~K}$. Iodine dosing was carried out in situ using an electrochemical source based on electrolysis of silver iodide.

\section{RESULTS AND DISCUSSION}

Figure 2 shows the angle-resolved photoemission spectrum of $\mathrm{W}\{531\}^{S}$ using RCP and LCP light of photon energy $30 \mathrm{eV}$, utilizing the experimental geometry depicted in Fig. 1. A number of features are clearly evident from the spectra. First and most importantly, the spectra are markedly different signifying that strong dichroic effects are obtained from chiral surfaces. A common secondary electron tail is observed in both spectra, in accordance with previous findings. ${ }^{17}$ This phenomenon was attributed in earlier work to multiplescattering processes. According to Schönhense and Hermes, ${ }^{17}$ such electrons " ... have no 'memory' of the angular distribution of the primary electrons. Their intensity 


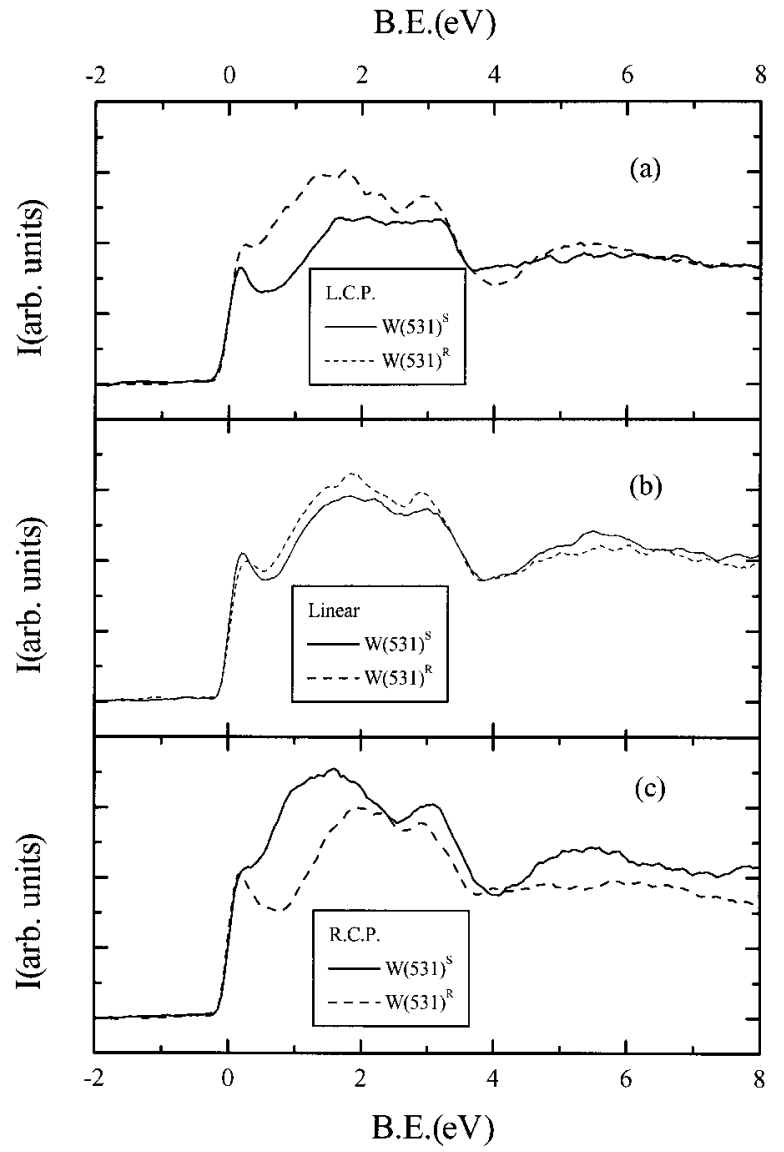

FIG. 3. Comparison of the valence-band photoemission spectra of $\mathrm{W}\{531\}^{R}$ and $\mathrm{W}\{531\}^{S}$ using (a) left circularly polarized light, (b) linearly polarized light, and (c) right circularly polarized light $\left(\theta=18^{\circ}, \phi=45^{\circ}\right.$, and $\left.\alpha=90^{\circ}\right)$. All the spectra have been smoothed, and the secondary electron tails subtracted.

is essentially given by the total photocurrent which cannot display a CD effect." Another point we wish to emphasize, and which we believe has not been highlighted in previous work, is the behavior of the tungsten surface state or resonance close to the Fermi energy of the valence band. This sharp peak (identified as a surface feature by its narrowness and its sensitivity to surface contamination and surface disorder) also displays a strong dichroic effect, which must originate in initial states confined solely to the surface atoms. Finally, a comment concerning the magnitude of the measured circular dichroism. It was stated in Sec. I that classical dichroic effects are small, whereas CDAD asymmetries may reach as much as $70-80 \%$. Since we ensured that $\vec{q}, \vec{k}$, and $\vec{n}$ were coplanar in our experimental geometry, this suggests that large dichroic effects are operative associated either with the intrinsic chirality of the surface or that some angular distribution effect is still operational. Figure 3 shows typical data obtained from $\mathrm{W}\{531\}^{R}$ and $\mathrm{W}\{531\}^{S}$ using RCP and LCP and linearly polarized (LP) light, following subtraction of the secondary electron tail and smoothing of the data. Taking first the spectra corresponding to LP light, in contrast to our expectations, there are photoemission differences between the $\mathrm{W}\{531\}^{R}$ and $\mathrm{W}\{531\}^{S}$ surfaces. We ascribe these spectral differences to experimental artifacts such as a greater degree of surface roughness associated with the $\mathrm{W}\{531\}^{R}$ plane relative to the $\mathrm{W}\{531\}^{S}$ plane. The $R$ surface directly faced the tungsten filament used to heat the crystal via electron emission. At the end of the experiment, visual inspection of the $R$ face revealed signs of surface imperfections, while the enantiomeric $S$ face retained a high quality. Clearly, the difference in the $R$ - and $S$-surface spectra with LP light represents a limit to the experimental precision attainable in the value of $A^{C D A D}$ (when using circularly polarized light), since it is much larger than any crystal alignment error in the calibration of the degree of helicity of the RCP and LCP photon flux. Based on the LP data for the tungsten $d$-band emission, a 5\% error in $A^{C D A D}$ is estimated, associated with a greater or lesser concentration of surface defects on opposite sides of the crystal, and not directly attributable to chiral effects.

Taken together, it is seen that the spectra using circularly polarized (CP) radiation, and $R$ and $S$ surfaces display a classical diasteromeric result in that

$$
\begin{aligned}
& W\{531\}^{R}+\mathrm{LCP} \equiv W\{531\}^{S}+\mathrm{RCP}, \\
& W\{531\}^{R}+\mathrm{RCP} \equiv W\{531\}^{S}+\mathrm{LCP},
\end{aligned}
$$

whereas

$$
\begin{aligned}
& W\{531\}^{R}+\mathrm{LCP} \neq W\{531\}^{R}+\mathrm{RCP}, \\
& W\{531\}^{S}+\mathrm{LCP} \neq W\{531\}^{S}+\mathrm{RCP} .
\end{aligned}
$$

It should be mentioned in this context that chiral single crystals provide a unique quantitative means of calibrating the relative amounts of circular polarization in the incident radiation, and that the relationships outlined above should always apply irrespective of CD or CDAD effects for equal degrees of left and right circular polarizations. Hence for "perfect" $R$ and $S$ crystal surfaces not subject to electron beam damage, the photoemission spectra of an $R$-surface/ LCP light combination should overlap with that of the $S$-surface /RCP light. Using LP radiation, electron photoemission from both the rear and the front faces of the crystal should also overlap.

Figure 4 shows the result of applying a peak deconvolution program to the smoothed data using five Gaussian peaks of fixed binding energy and peak width, in order that $A^{C D A D}$ may be estimated for different regions of the spectrum. This was the minimum number of peaks found necessary to fit the raw data. The fixed values of binding energy and peak width were obtained iteratively from the photoemission data for the four combinations of CP light and the chiral crystal surface. Once these parameters were obtained, the peak areas were measured. It is recognized that this was a purely empirical procedure although, remarkably, once the peak positions and widths were obtained as constants for the four spectra (using the iteration described previously) very little variation in the ultimate curve fit and peak areas was found. Peaks $B, D$, and $E$ were found to exhibit similar, small $A^{C D A D}$ values of $2 \%$. Far larger values of asymmetry parameter were found for peaks $A$ (the surface state or resonance) and $C$. Values of $A^{C D A D}$ calculated for these peaks were as follows: 


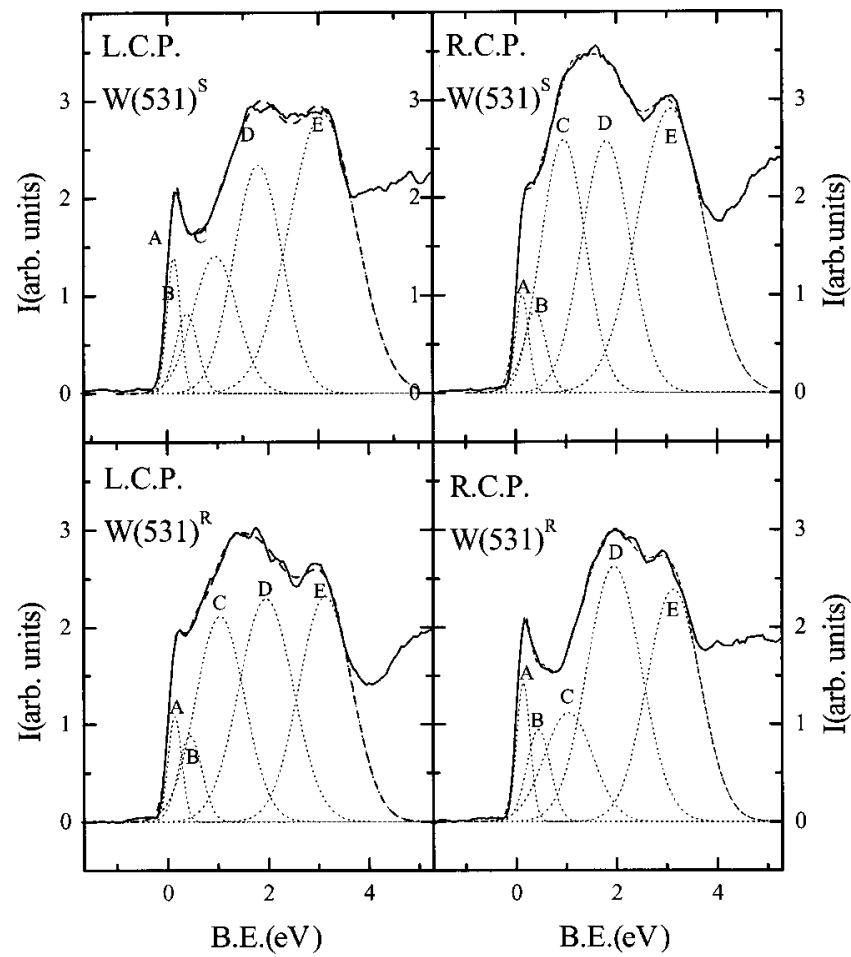

FIG. 4. A five-peak deconvolution of the valence band of $\mathrm{W}\{531\}^{R}$ and $\mathrm{W}\{531\}^{S}$ with left and right circularly polarized light of $30 \mathrm{eV}$. The dashed lines indicate the fit to the data.

$$
\begin{aligned}
& A^{C D A D}(\text { peak } A)=15 \% \pm 5 \%, \\
& A^{C D A D}(\text { peak } C)=30 \% \pm 5 \% .
\end{aligned}
$$

Such large values of the asymmetry parameter cannot be ascribed solely to classical CD effects. Therefore, as an experimental control, photoemission experiments under identical conditions to those for the $\mathrm{W}\{531\}$ sample were performed on the close-packed, achiral $\mathrm{W}\{110\}$ and $\mathrm{O} / \mathrm{W}\{110\}$, surfaces (Figs. 5 and 6 , respectively). Although $\vec{n}, \vec{q}$, and $\vec{k}$

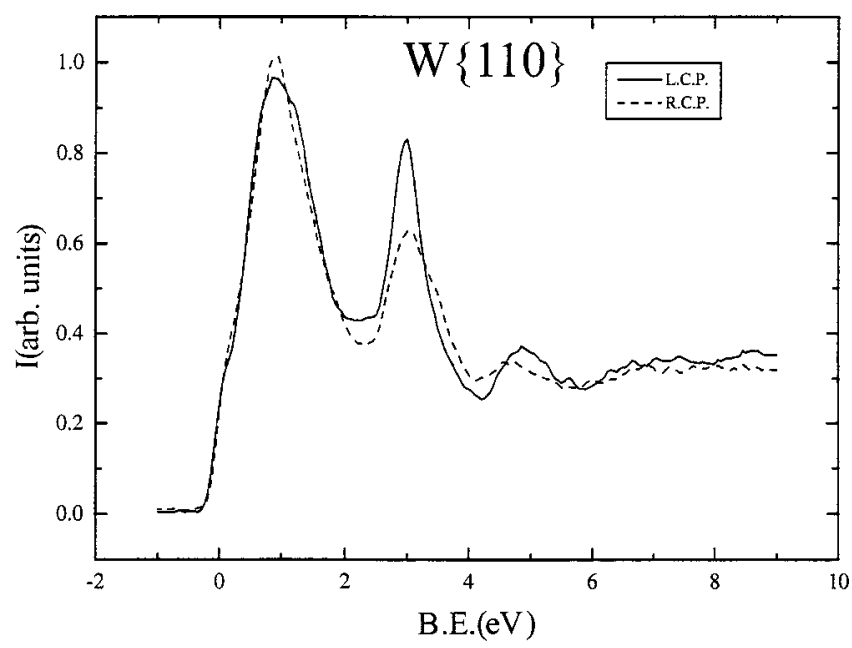

FIG. 5. Valence-band photoemission spectra of achiral W $\{110\}$ taken with right and left circularly polarized light (photon energy 30 $\mathrm{eV}, \theta=18^{\circ}, \phi=45^{\circ}$, and $\alpha=90^{\circ}$ ).

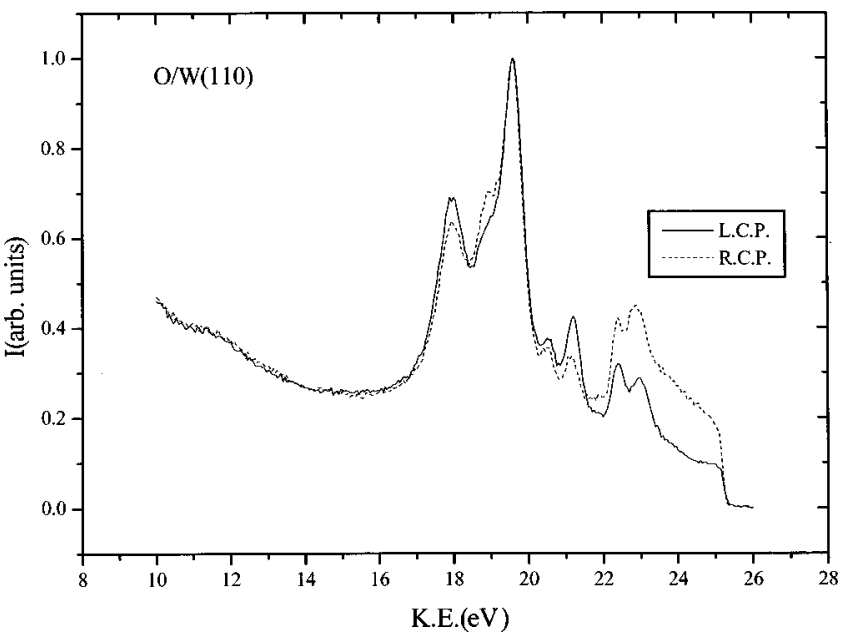

FIG. 6. Valence-band photoemission spectra of achiral W $\{110\}$ taken with right and left circularly polarized light (photon energy 30 $\mathrm{eV}, \phi=18^{\circ}, \theta=45^{\circ}$, and $\alpha=90^{\circ}$ ).

were coplanar, strong dichroic effects are apparent. Since there is no intrinsic chirality associated with a W $\{110\}$ surface, the circular dichroism must be associated with the experimental geometry in some way, i.e., an angular distribution effect is still present in the photoemission spectra. The origin of this must be addressed. The explanation lies in the fact that photoelectron scattering is important and that in our (fixed) experimental geometry the detected photoelectrons do not arise from a mirror plane of the surface where scattering induced CD would be zero. Rather $\phi$ (the azimuthal angle relative to the [110] direction) was at a fixed angle of $18^{\circ}$. In fact Schönhense et al. already reported such effects in the valence-band photoemission from $\mathrm{C}\{0001\}$ and $\operatorname{Pd}\{111\}$ using photon energies $<25 \mathrm{eV}$, although in these cases, $\vec{n}, \vec{q}$, and $\vec{k}$ were not coplanar. ${ }^{7}$ The azimuthal angle $\phi$ was not specified in their work, and hence it is not clear whether $\vec{n}$ and $\phi$, or both, determine the handedness of the system and hence any dichroism. Since W\{110\} asymmetry factors of a similar order of magnitude to those for the $\mathrm{W}\{531\}$ data set could be generated for a coplanar arrangement of $\vec{n}, \vec{q}$, and $\vec{k}$, not corresponding to a mirror plane of the surface, we conclude that final-state effects dominate angle-resolved photoemission from both chiral and achiral substrates using CP light. It is interesting that even when surface diffraction is minimized, as, for example, when considering emission from a surface state or resonance into the vacuum, there results a strong dichroic effect (15\%). Interference between direct emission from the surface state into vacuum and scattered electrons from the tungsten substrate generate these large dichroic effects. Theoretical calculations of the magnitude of $\mathrm{CD}$ under these experimental conditions are presently being undertaken.

How, therefore, can one decouple final-state CDAD effects from classical CD associated with chiral kinks? There are a number of ways of doing this which will be the subject of a future publication. However, in the present context, it is evident from previous work that if the surface contains a mirror plane of symmetry and $\vec{k}$ and $\vec{q}$ are contained within 


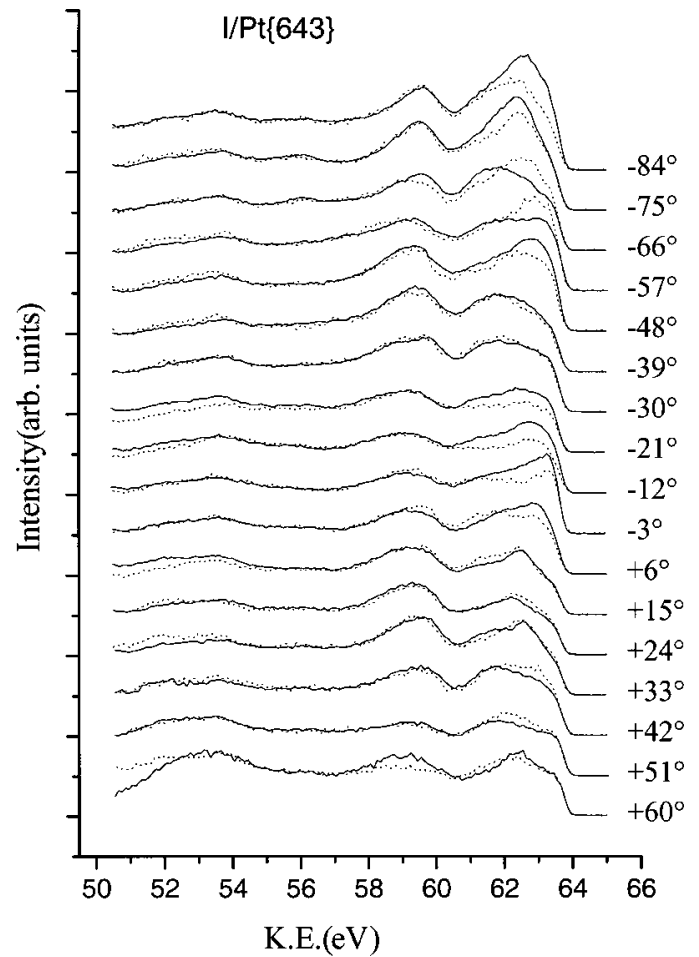

FIG. 7. Valence-band photoemission spectra ( $70 \mathrm{eV}$ photons) of iodine-dosed $\operatorname{Pt}\{643\}$ as a function of azimuthal angle, $\phi$; solid line taken with RCP light dotted line with LCP light.

it, then the scattering induced $\mathrm{CD}$ will be zero. ${ }^{9}$ Chiral surfaces do not contain a mirror plane of symmetry, they are therefore expected to give rise to dichroism at all azimuthal angles. Conversely, if a surface gives rise to dichroism at all azimuthal angles, it must be chiral. To test this hypothesis, we examined the azimuthal dependence of the photoemission spectrum of the $(\sqrt{3} \times \sqrt{3}) R 30^{\circ}$ iodine phase of $\operatorname{Pt}\{643\}^{S}$ using CP radiation. Iodine overlayers are known to pacify platinum surfaces toward adsorption of residual gas atoms, ${ }^{18}$ and as we did not want to repeatedly clean the surface during the measurement, the iodine phase provided a convenient way of maintaining a well-defined surface of unchanging, inherent chirality. It is also known from recent scanning tunneling microscopy studies ${ }^{18}$ that iodine atoms adsorbed at room temperature do not induce reconstruction in either terrace or step sites on platinum.

Figure 7 shows a family of valence band photoemission curves for $\mathrm{I} / \mathrm{Pt}\{643\}^{S}$ using $70 \mathrm{eV}$ photons and a scattering geometry, whereby $\vec{q}, \vec{n}$, and $\vec{k}$ are coplanar. The azimuthal angle $\phi$ is defined relative to the [011] direction in the three atom wide $\{111\}$ terraces constituting the kinked $\operatorname{Pt}\{643\}$ plane (in microfacet notation $\{643\} \equiv 3\{111\} \times\{310\}$; see Fig. 8). It is clear from Fig. 7 that although a strong dependence of spectral asymmetry as a function of azimuthal angle is observed, at $\phi=-30^{\circ}$ (the scattering plane along the $[\overline{1} 2 \overline{1}]$ direction of the $\{111\}$ terrace) and to a lesser extent

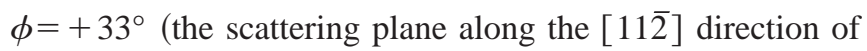
the $\{111\}$ terrace), the dichroism does indeed approach zero, as signified by the almost perfect overlap of the spectra using LCP and RCP light. The $[\overline{1} 2 \overline{1}]$ and $[12 \overline{2}]$ directions are

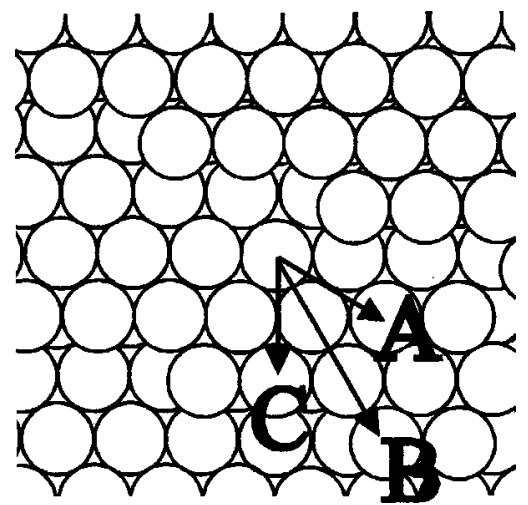

FIG. 8. Ball model of the $\operatorname{Pt}\{643\}$ surface showing scattering directions relative to $\{111\}$ terrace sites. $A=\langle\overline{1} 21\rangle, B=\langle 01 \overline{1}\rangle$, and $C=\langle 11 \overline{2}\rangle$.

significant, since they constitute mirror planes of the $\{111\}$ terrace. We speculate that a reasonable explanation of this behavior is that scattering geometries containing these bulk crystal mirror planes may lead to cancellation of CDAD effects, i.e., the "residual" $\{111\}$ character of the chiral $\{643\}$ surface is sufficiently strong that it effectively facilitates decoupling of dichroic effects associated with the chiral experimental geometry from the measurement. Nonetheless, the asymmetry does not go exactly to zero within the precision of our experiment.

Figure 9 shows an expanded version of the $\phi=-30^{\circ}$ data. The intensity of the spectrum using RCP light was always slightly smaller than for LCP light in the vicinity of the two peaks at kinetic energies of 59.5 and $62.5 \mathrm{eV}$. However, because of the noise level on the data, in particular that corresponding to LCP light, the value of $A$ for both peaks is estimated to be $2 \% \pm 1 \%$. Since dichroism should not appear in photoemission for $\phi= \pm 30^{\circ}$ with $\vec{n}, \vec{q}$, and $\vec{k}$ all coplanar at these "pseudo" mirror planes, we tentatively ascribe this residual level of $2 \%$ asymmetric behavior to the classical circular dichroism in photoemission exhibited by the kinked surface. Unfortunately, it was not possible to repeat the mea-

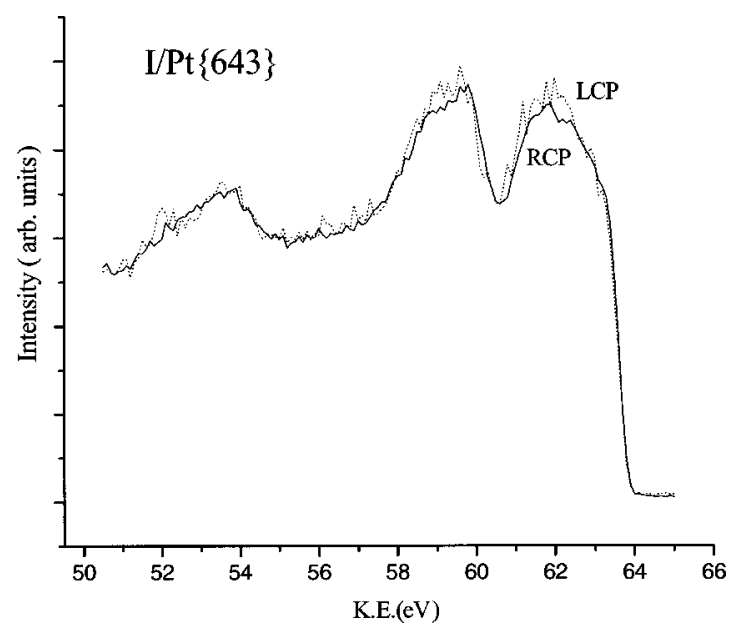

FIG. 9. An expansion of the I/Pt $\{643\}$ valence-band photoemission spectra (70 eV photon energy) at $\phi=30^{\circ}$ in Fig. 7. 
surement for the $\operatorname{Pt}\{643\}^{R}$ surface to test for the diastereomeric result, since the crystal sample was a "Clavilier bead," 16 with only one face accessible. Additional experimentation is planned to validate (or otherwise) this finding.

\section{CONCLUSIONS}

Valence-band photoemission data from clean and adsorbate-covered, chiral, and kinked single-crystal metal surfaces have been collected. Because the magnitude of dichroic effects observed far exceeds any reasonable estimate of classical CD attributable to chiral kink photoemission, it is concluded that final-state photoelectron effects are dominating the spectra even for electron emission from states localized in the metal surface. The residual $\{111\}$ character of the chiral I/Pt $\{643\}^{S}$ surface also provides a mechanism for attenuation of dichroic effects, since scattering geometries containing mirror planes of the $\{111\}$ terrace lead to very small

*Email address: ATTARD@CARDIFF.AC.UK

${ }^{1}$ A. Ahmadi, G. A. Attard, J. M. Feliu, and A. Rodes, Langmuir 15, 2420 (1999).

${ }^{2}$ B. Ritchie, Phys. Rev. A 12, 567 (1975); 13, 1411 (1976); 14, 359 (1976); 14, 1396 (1976).

${ }^{3}$ N. Chandra, Phys. Rev. A 39, 2256 (1989).

${ }^{4}$ L. D. Barron, Molecular Light Scattering and Optical Activity (Cambridge University Press, Cambridge, 1982).

${ }^{5}$ N. A. Cherepkov, Chem. Phys. Lett. 87, 344 (1982).

${ }^{6}$ C. Westphal, J. Bansmann, M. Getzlaff, and G. Schönhense, Phys. Rev. Lett. 63, 151 (1989).

${ }^{7}$ G. Schönhense, C. Westphal, J. Bansmann, M. Getzlaff, J. Noffke, and L. Fritche, Surf. Sci. 251/252, 132 (1991).

${ }^{8}$ C. Westphal, J. Bansmann, M. Getzlaff, and G. Schönhense, J. Electron Spectrosc. Relat. Phenom. 52, 613 (1990).

${ }^{9}$ R. X. Ynzunza, H. Daimon, F. J. Gardia de Abajo, J. Morais, R. Denecke, J. B. Kortright, Z. Hussain, M. A. Van Hove, and C. S. Fadley, J. Electron Spectrosc. Relat. Phenom. 106, 7 (2000). values of $\mathrm{A}(=2 \% \pm 1 \%)$. Within our experimental precision, we tentatively assign this low level of asymmetry to the classical CD of the chiral kink sites.

\section{ACKNOWLEDGMENTS}

We would like to acknowledge the financial support of the EPSRC for the award of beamtime on the SRS and for a studentship to DW (EPSRC Grant No. M65724). G.A.A. would also like to express his gratitude to Professor C. S. Fadley for useful discussions during the preparation of the paper. In addition, we acknowledge Dr. Mike MacDonald for his help and for his continued interest in the experimental work reported in this paper. Technical support was gratefully received from Dr. S. Patel, A. Hannah, and Dr. A. Smith. Finally, our thanks are particularly due to I. Sutton and A. Alexander who solved, with great elegance and speed, a number of electronics problems.

${ }^{10}$ H. Daimon, T. Nakatani, S. Imada, S. Suga, Y. Kagoshima, and T. Miyahara, Jpn. J. Appl. Phys. 32, L1480 (1993).

${ }^{11}$ G. E. Fecher, Europhys. Lett. 29, 605 (1995).

${ }^{12}$ P. P. Manning, N. J. Clague, I. W. Kirkman, F. M. Quinn, and P. J. Hicks, Nucl. Instrum. Methods Phys. Res. A 392, 345 (1997).

${ }^{13}$ P. P. Manning, N. J. Clague, A. Hannah, F. M. Quinn, D. Teehan, and P. J. Hicks, J. Synchrotron Radiat. 5, 848 (1998).

${ }^{14}$ M. MacDonald, Station manual: http://srs.dl.ac.uk/XUV-VUV/ 3.3manual.htm

${ }^{15} \mathrm{M}$. MacDonald (unpublished).

${ }^{16}$ J. Clavilier, D. Armand, S. G. Sun, and M. Petit, J. Electroanal. Chem. 205, 267 (1986).

${ }^{17} \mathrm{G}$. Schönhense and J. Hormes in VUV and Soft X-ray Photoionization, edited by Uwe Becker and David A. Shirley (Plenum Press, New York, 1996).

${ }^{18}$ E. Herrero, J. M. Orts, A. Aldaz, and J. M. Feliu, Surf. Sci. 440, 259 (1999). 